J. Clin. Chem. Clin. Biochem.

Vol. 15,1977 , pp. 593-602

\title{
Der Analysen-Leerwert beim Radioimmunoassay: Einflüsse auf die unspezifische Antigen-Bindung und ihr Effekt auf die Richtigkeit der Ergebnisse bei der Insulin-Bestimmung
}

\author{
Von $A$. Dwenger, P. Tost und I. Trautschold \\ Institut für Klinische Biochemie.der Medizinischen Hochschule Hannover
}

(Eingegangen am 21. Januar/7. April 1977)

Zusammenfassung: Die unspezifische Bindung hat als Analysen-Leerwert bei radioimmunologischen Testverfahren eine außerordentliche Bedeutung für die Qualitätskontrolle. Da Proben- und Standard-Antigen zumeist nicht in identischen Medien vorliegen, das Medium seinerseits aber die Höhe von Antigen-Antikörper-Bindung und unspezifischer Bindung beeinflußt, kann daher ein Effekt auf die Richtigkeit der Ergebnisse nicht ausgeschlossen werden. Anhand der Insulin-Bestimmung kann demonstriert werden, daß es bei Adsorptions-Trenntechniken (dextranbeladene Aktivkohle, Ionenaustauscher) zu einem Anstieg der unspezifischen Bindung mit zunehmendem Alter des markierten Antigens kommt, bei chemischen Präzipitations-Trenntechniken (Natriumsulfit, Polyethylenglycol 4000) dagegen nicht. Aus einem Vergleich der für unterschiedliche Antigen-Konzentrationen ohne Antiserum ermittel ten unspezifischen Bindungen (UB) und derjenigen mit Antiserum und Antigen-Uberschuß (UB') geht hervor, daß UB und $\mathrm{UB}^{\prime}$ identisch sind und daß UB unabhängig vom Antigen/Antikörper-Verhältnis konstant ist. Die Antigen-Antikörper-Bindungen der Standards können daher durch UB oder UB' korrigiert werden. Aufgrund der Konstanz von UB ist das vereinfachte Verfahren zulässig, UB für einen Standard zu ermitteln und zur Korrektur der weiteren Standards zu verwenden.

Anhand der Untersuchung von Diabetikerseren wird gezeigt, daß Serumproben, die Antikörper oder Autoantikörper enthalten, mit Hilfe der individuellen unspezifischen Bindungen erkannt und dann von der Testauswertung ausgeschlossen werden können, wobei Unterschiede zwischen UB und UB' Hinweise auf das Ausmaß der Teststörung geben.

Die Richtigkeit der Ergebnisse bei der Insulin-Bestimmung ist von der Summe der Einflüsse abhängig, die das Medium für die Standards; die Art der Trenntechnik und die Korrektur für die individuellen unspezifischen Bindungen auf den Test ausüben. In variabler Testdurchführung durch Kombination unterschiedlicher Medien für die Standards ( a) Pufferlösung mit Immunglobulin vom Rind, b) insulinarmes Humanserum, c) Pufferlösung mit Humanalbumin und Immunglobulin vom Rind) mit drei Trenntechniken (Aktivkohle/Dextran, Natriumsulfit, Polyethylenglycol 4000) und drei Verfahren zur Korrektur für die unspezifischen Bindungen werden die Ergebnisse aus WiederfindeVersuchen einander gegenübergestellt. Aus dem Vergleịch der Resultate wird abgeleitet, daß eine Korrektur für die individuelle unspezifische Bindung bei Standards und Proben notwendig ist.

\section{The analytical blank in radioimmunoassay.}

Factors influencing the unspecific binding of antigen and its effects on the accuracy of the results for the insulin assay

Summary: The use of unspecific binding as an analytical blank in radioimmunoassay is very important in quality control. Since the media of the sample and the standard are usually different, and since the medium influences the binding of the antigen to the antibody as well as unspecific binding of the antigen, an effect on the accuracy of the results may not be excluded.

For insulin radioimmunoassay it is demonstrated that separation techniques with adsorbents (dextran coated charcoal, ion exchange resin) cause an increase of unspecific binding depending on the age of the labeled antigen, whereas separation by chemical precipitation (sodium sulphite, polyethyleneglycol 4000) gives constant values for unspecific binding. Comparison of the unspecific binding evaluated by omission of antiserum (UB) in the test system for different antigen concentrations and the unspecific binding measured in the presence of antiserum and 
an excess of unlabeled antigen (UB') give identical results. $U B$ is constant for different antigen/antibody relations. Therefore UB as well as $U B^{\prime}$ may be used for correction of binding values of the standard curve. Furthermore the constancy of UB for all standards favours the simplified procedure for the determination of UB only for one value of the standards, preferably for zero binding, and for the correction of all standards with this value.

Serum samples from diabetics containing antibodies or serum samples containing autoantibodies may be recognized by the individual unspecific bindings and then eliminated from calculation; differences between UB and $\mathrm{UB}^{\prime}$ may indicate the extent of this interference.

There are complex influences on the accuracy of radioimmunologically determined serum insulin concentrations. These influences result from the medium for the standard curve, the separation technique and the correction for unspecific binding. As demonstrated by recovery experiments accuracy varies for all possible combinations of three media for the standard curve ( $a$, buffer solution with bovine immunglobulin, $b$, human serum with a low insulin concentration, c, buffer solution with human albumin and bovine immunglobulin) with three separation techniques (dextran coated charcoal, sodium sulphite, polyethyleneglycol 4000) and with three methods of correction for unspecific binding. From the results it is evident that correction for the individual unspecific binding is necessary.

\section{Einleitung}

Für radioimmunologische Bestimmungs-Verfahren ist charakteristisch, daß für nahezu jedes Antigen eine Reihe von Testaufbauten existiert, die sich in wesentlichen Teilschritten des Gesamt-Analysen-Ablaufs grundsätzlich voneinander unterscheiden. Trotz der allen Verfahren zugrundeliegenden komparativen Arbeitsweise zeigen sich in Ringversuchen $(1,2)$ und vergleichenden Untersuchungen von Testkombinationen erhebliche Unterschiede in den Ergebnissen. Einen Hinweis auf die unzureichende Standardisierung dieser Analytik liefert auch der offenkundige Mangel an Testverfahren-unabhängigen Normwerten oder Normbereichen. Es ist nicht auszuschließen, daß die allgemeine Vernachlässigung des Analysen-Leerwertes in der radioimmunologischen Analytik hiermit in engem Zusammenhange steht. Dem Problem von Leerwerten wird auch in dem Bericht der Internationalen AtomenergieOrganisation zur Standardisierung dieser Verfahren (3) durch die alleinige Empfehlung zur subtraktiven Korrektur der unspezifischen Bindung nur formale Bedeutung zugemessen. Demgegenüber haben Ekins \& Newman (4) bereits früher eine Abhängigkeit der unspezifischen Bindungen von den relativen Antigen/Antikörper-Konzentrationen diskutiert und auf eine für die Standardkurve bereichsabhängige Differenzierbarkeit hingewiesen.

In zunehmendem Maße mitgeteilte Befunde, die ein von Erfahrung und Norm abweichendes Verhalten bei radioimmunologischen Tests beschreiben (5-20), zeigen, daß den Test beeinflussende Faktoren existieren, von denen sich einige mit einer ungewöhnlichen Verhaltensweise der unspezifischen Bindungen erklären lassen $(9,12-20)$.

Unterschiede in den Leerwerten für Standards und Proben können bei vergleichenden Analysen-Verfahren einen Einfluß auf die Richtigkeit der Ergebnisse ausüben, sofern nicht eine individuelle Korrektur für den Leerwert zur Beseitigung dieser Unterschiede beiträgt.
Für radioimmunologische Verfahren ist wenig darüber bekannt, wodurch unspezifische Bindungen beeinflußt werden und wie sich ihr Einfluß auf die Ergebnisse auswirkt. Einer der Gründe, daß unterschiedlich hohe unspezifische Bindungen für Standards und Proben beob. achtet werden, ist auf ihre Medien-Differenzen zurückzuführen. Insbesondere gilt dies für die Bestimmung endogener Antigene, da aufgrund des Mangels an antigenfreien Seren ein artifizielles Medium für die Standards verwendet werden muß. Hierdurch zeigen sowohl die Antigen-Antikörper-Bindungen als auch die unspezifischen Bindungen medienabhängig jeweils unterschiedliche Höhen. Es erscheint daher erforderlich, diese Unterschiede im jeweiligen radioimmunologischen Versuchsaufbau zu kennen und zu untersuchen, ob die Korrektur für die individuellen unspezifischen Bindungen geeignet ist, die Richtigkeit der Ergebnisse zu verbessern. Im radioimmunologischen Test lassen sich unspezifische Bindungen in Reaktionsansätzen ermitteln, die kein Antiserum enthalten (UB) oder in Reaktionsansätzen, denen in Gegenwart von Antiserum ein Antigen-Überschuß zugesetzt wird (UB').

Da in diesem Zusammenhange bisher keine vergleichenden Untersuchungen bekannt sind, werden exemplarisch anhand eines radioimmunologischen Tests solche Faktoren beschrieben, die einen Einfluß auf die unspezifische Bindung haben und der Einfluß der unspezifischen Bindung auf die Richtigkeit der Ergebnisse wird untersucht. Die Wahl der Insulin-Bestimmung als Modell resultiert aus der relativen Häufigkeit dieses Tests, der Anwendbarkeit unterschiedlicher Prinzipien zur Trennung des freien vom gebundenen Antigen, der Möglichkeit, die durch einen endogenen Antikörper bedingte Teststörung anhand der unspezifischen Bindungen beschreiben zu können und aus der für endogene Antigene typischen Schwierigkeit, ein natives antigenfreies Serum zur Herstellung von Standards verwenden zu können und der hieraus folgenden Notwendigkeit zur Untersuchung verschiedener allgemein-zugänglicher Medien. 
Grundsätzlich scheinen drei Komponenten die Höhe der unspezifischen Bindung zu beeinflussen: die Reinheit des markierten Antigens, das der Trenntechnik zugrundeliegende Prinzip und die Zusammensetzung des Mediums, in dem Proben- bzw. Standard-Antigen vorliegen.

Fragestellungen und Versuchsaufbauten gliedern sich in vier Teile:

1. Welche Einflüsse haben die Reinheit des markierten Antigens und ein unterschiedliches Prinzip der Trenntechnik auf die Antigen-Antikörper-Bindung und die unspezifische Bindung UB? ${ }^{*}$

2. Sind die nach zwei Verfahren zu ermittelnden unspezifischen Bindungen UB und UB' für Standards identisch und ist die Höhe von UB vom Antigen/Antikörper-Verhältnis abhängig?

3. Kann die unspezifische Bindung ein Indikator zur Erkennung von Teststörungen sein, wie sie $z$. B. durch die Anwesenheit eines endogenen Antikörpers in einer Serumprobe verursacht werden?

4. Welchen Einfluß hat die Korrektur für die individuellen unspezifischen Bindungen auf die Richtigkeit der Ergebnisse?

Zur Untersuchung dieser Fragestellung wird die Wiederfindung von Insulin in Serum anhand von neun Kombinationen im Testaufbau (3 Medien für die Standards, 3 Trenntechniken) in Abhängigkeit von der Korrektur für die individuellen unspezifischen Bindungen UB und UB' von Standards und Proben beschrieben.

\section{Material und Methodik}

Lösungen, Proben

Pufferlösung 1: Barbital-Natrium 7,13 mmol/1; Natriumacetat $11,8 \mathrm{mmol} / \mathrm{l}$; Natriumchlorid $138 \mathrm{mmol} / \mathrm{l}$; Natriumazid $15,4 \mathrm{mmol} / \mathrm{l}$; Serumalbumin vom Rind $5 \mathrm{~g} / \mathrm{l}$; pH-Wert 7,4.

Pufferlösung 2: Pufferlösung $1 \mathrm{mit} 20 \mathrm{~g} / 1 \mathrm{Immunglobulin} \mathrm{vom}$ Rind.
Pufferlösung 3: Pufferlösung 1 ohne Serumalbumin vom Rind. Tracerlösung: ${ }^{125} \mathrm{~J}$-markiertes Schweineinsulin mit ciner spezifischen Radioaktivität von $6,48 \mathrm{GBq} / \mathrm{mg}$. Hieraus wcrden Lösungen mit 5000-80000 Imp/min $100 \mu$ Pufferlösung 1 hergestellt (Versuchsteile 1-3) bzw. wird eine Lösung mit etwa $10000 \mathrm{Imp} / \mathrm{min} \cdot 50 \mu \mathrm{l}$ Pufferlösung 1 hergestellt (Versuchsteil 4).

Antikörperlösung: Anti-Schweineinsulin-Serum vom Meerschweinchen ${ }^{1}$ ) wird mit Pufferlösung 1 verdünnt; bei einer Antiserum-Endverdünnung von 1:750000 im Test werden unter Verwendung von Aktivkohle/Dextran als Trennmittel in 20 Stunden bei $4^{\circ} \mathrm{C}$ und bei Einsatz von $8000 \mathrm{Imp}$./min pro Test 50\% Tracer gebunden.

Standard-Insulin: Schweine-Insulin; eine definierte Menge Standard-Insulin wird in $0,03 \mathrm{~mol} / 1$ Salzsäure gelöst und mit Pufferlösung 1 auf die erforderlichen Standard-Konzentrationen verdünnt.

Standards (Versuchsteil 4): Aus einer Insulinlösung mit $100 \mathrm{E} / \mathrm{l}$ werden durch Verdünnung mit drei Medien $(\mathrm{a}, \mathrm{b}, \mathrm{c})$ Standards für einen Konzentrations-Bereich von 0 bis $320 \mathrm{mE} / 1$ hergestellt.

Medium a: Pufferlösung 2

Medium b: insulinarmes Humanserum Fluinorm $N$ ( $\leqslant 2 \mathrm{mE} / 1$ Insulin)

Medium c: Pufferlösung 3 mit $50 \mathrm{~g} / 1$ Humanalbumin und $20 \mathrm{~g} / \mathrm{l}$ Immunglobulin vom Rind.

Proben: Dem Serum eines Blutspenders wird Standard-Insulin zugesetzt, wobei neun Serumproben mit den Konzentrationen $0,10,20,30,40,50,60,70$ und $80 \mathrm{mE} / 1$ zugesetztes Insulin resultieren.

\section{Verwendete Reagenzien}

E. Merck: 5,5-Diethylbarbitursäure Natriumsalz, zur Analyse; Natriumacetat krist., zur Analyse; Natriumchlorid krist., zur Analyse; Natriumazid; Natriumsulfit, wasserfrei, rein; Aktivkohle zur Analyse.

Serva: Albumin aus Rinderserum lyophil. rein, $92 \% \mathrm{~min}$.

Behringwerke AG: Humanserum zur Richtigkeitskontrolle Fluinorm N; Immunglobulin vom Rind, reinst; Humanalbumin, trocken, reinst.

Farbwerke Hoechst AG: ${ }^{125} \mathrm{~J}-\mathrm{Sch}$ weine-Insulin; Amberlite CG $400 \mathrm{I}$ in Kapseln, ca. $250 \mathrm{mg} / \mathrm{Kapsel}$.

Novo: Insulin vom Schwein, $10 \times$ krist.; $1 \mathrm{mg}=25 \mathrm{I}$. E.

Fluka AG: Dextran MG 80000 purum.

Merck-Schuchardt: Polyethylenglycol 4000 zur Synthese.

1) Das Anti-Schweineinsulin-Serum stammt von Dr. J. R. Williamson, Johnson Research Foundation, Philadelphia, USA.

Testzụsammensetżungen für die Versuchsteile $1-3$

\begin{tabular}{|c|c|c|c|c|c|c|c|c|}
\hline & \multicolumn{4}{|c|}{ Standardkurve } & \multicolumn{4}{|c|}{ Proben $a-d$} \\
\hline . & $\mathrm{UB}_{0}$ & UB' $^{\prime}$ & $\mathrm{B}_{0}$ & $\mathrm{UB}_{\mathrm{St}}$ & $\mathrm{B}_{\mathrm{St}}$ & UB & $\mathrm{UB}^{\prime}$ & B \\
\hline $\begin{array}{l}\text { Tracerlösung }[\mu \mathrm{l}] \\
\text { (8000 Imp./min } \cdot 100 \mu l \text { bżw. variąbel) }\end{array}$ & 100 & 100 & 100 & 100 & 100 & 100 & 100 & 100 \\
\hline Pufferlösung $2[\mu]\rceil$ & 100 & 100 & 100 & 100 & 100 & - & - & - \\
\hline Pufferlösung $1[\mu !]$ & 300 & 100 & 200 & 200 & 100 & 300 & 100 & 200 \\
\hline Standards $[\mu \mathrm{\mu l}](5-320 \mathrm{mE} / \mathrm{l})$ & - & - & - & 100 & 100 & - & - & - \\
\hline Lösung mit Antigen-Überschuß [ $\mu l]$ & - & 100 & - & - & - & - & 100 & - \\
\hline Proben $[\mu \mid \mu]$ & - & - & - & - & - & 100 & 100 & 100 \\
\hline Antịkörperlösung [ $\mu 1](1: 130000)$ & - & 100 & 100 & - & 100 & - & 100 & 100 \\
\hline
\end{tabular}


Versuchsteil 1: Bestimmung von $\mathrm{B}_{0}$ und $\mathrm{UB}_{0}$ mit unterschiedlich altem Tracer-Insulin.

Versuchsteil 2: Bestimmung von $\mathrm{UB}_{0}, \mathrm{UB}^{\prime}$ und $\mathrm{B}_{0}$ mit konstanten $(8000 \mathrm{Imp} . / \mathrm{min})$ sowie variablen $(5000-80000 \mathrm{Imp} . / \mathrm{min})$ Tracermengen; die Konzentration der Lösung mit Antigen-Uberschuß beträgt $500 \mathrm{E} / 1$; ferner werden $\mathrm{UB}_{\mathrm{St}}$ und $\mathrm{B}_{\mathrm{St}}$ bestimmt.

Versuchsteil 3: Zusammensetzung der im Schema angegebenen Reaktionsansätze unter Verwendung einer Lösung mit AntigenÜberschuß von $100 \mathrm{E} / 1$ zur Ermittlung von $\mathrm{UB}^{\prime}$ bei Standards und Proben a-c; für die Reaktionsansätze mit der Probe $d$ werden Lösungen mit Antigen-Überschuß von 30-1000 E/l verwendet.

Probe a: Fluinorm N

Probe b: Diabetikerserum $S_{1}$

Probe c: Diabetikerserum $S_{2}$

Probe d: Diabetikerserum $\mathrm{S}_{3}$

Testbedingungen: Inkubationszeit 20 Stunden Inkubationstemperatur $4{ }^{\circ} \mathrm{C}$.

Testzusammensetzungen für den Versuchsteil 4

\begin{tabular}{|c|c|c|c|c|c|c|c|c|}
\hline & & \multicolumn{4}{|c|}{ Standardkurve } & \multicolumn{3}{|c|}{ Proben 1-9 } \\
\hline & & $\mathrm{UB}_{0}$ & UB' & $\mathbf{B}_{0}$ & $\mathrm{BSt}$ & UB & $\mathrm{UB}^{\prime}$ & B \\
\hline Tracerlösung $[\mu]]$ & & 50 & 50 & 50 & 50 & 50 & 50 & 50 \\
\hline Medium a, b oder $c[\mu]]$ & & 50 & 50 & 50 & - & - & - & - \\
\hline Standards in a, b oder $\mathrm{c}[\mu \mathrm{l}](5-320 \mathrm{mE} / \mathrm{l})$ & & - & - & - & 50 & - & $\dot{-}$ & - \\
\hline Proben $[\mu l]$ & ' & - & - & - & - & 50 & 50 & 50 \\
\hline Lösung mit Antigen-Überschuß $[\mu l]$ & & - & 50 & - & - & - & 50 & - \\
\hline Pufferlösung $1[\mu \mid]$ & & 150 & 50 & 100 & 100 & 150 & 50 & 100 \\
\hline Antikörperlösung [ $\mu 1](1: 150000)$ & & - & 50 & 50 & 50 & - & 50 & 50 \\
\hline
\end{tabular}

Die Reak tionsansätze für die Standardkurven werden jeweils für die Medien a, $b$ und $c$ vorbereitet.

Testbedingungen: Inkubationszeit 20 Stunden Inkubationstemperatur $4{ }^{\circ} \mathrm{C}$

\section{Testdurchführung}

Nach Vorbereitung und Inkubation der Reaktionsansätze werden in den Versuchsteilen 1-3 je $1000 \mu \mathrm{l}$, im Versuchsteil 4 je $500 \mu \mathrm{l}$ Trennmittel zudosiert.

A. Aktivkohle/Dextran-Suspension; Endkonzentration 12,5 g/1/ $1,25 \mathrm{~g} / \mathrm{l}$ bei Reaktionsansätzen in den Versuchsteilen 1 und 4

B. Natriumsulfit; Endkonzentration $153 \mathrm{~g} / \mathrm{l}$ bei Reaktionsansätzen in den Versuchsteilen 1, 2 und 4

C. Polyethylenglycol 4000; Endkonzentration $175 \mathrm{~g} / \mathrm{l}$ bei Reaktionsansätzen in den Versuchsteilen 1,3 und 4

D. Amberlite CG 400 I-Suspension; Endkonzentration $170 \mathrm{~g} / \mathrm{l}$ Testansatz bei Reaktionsansätzen im Versuchsteil 1

Nach Zugabe der Trennmittel wird bei A. 1 Minute bei Raumtemperatur inkubiert, bei $B$. und $C$. jeweils 5 Minuten und bei D. 10 Minuten. Anschließend wird für 4 Minuten bei $12000 \mathrm{~g}$ zentrifugiert und ein Aliquot des Überstandes abgenommen. Die Radioaktivitäten werden in beiden Anteilen gemessen.

Berechnungen für die Versuchsteile $1-3$

Für jeden Reaktionsansatz wird die prozentuale Bindung des $125 \mathrm{~J}$-Insulins berechnet:

$$
\% B=\left(\frac{\text { Imp. } / \min _{\ddot{U}} \cdot F}{I m p . / \min _{\ddot{U}}+\text { Imp./mins }}\right) \cdot 100
$$

für Trenntechniken A. und D.

$$
\% \mathrm{~B}=\left(1-\frac{\mathrm{Imp} . / \mathrm{min} \ddot{U} \cdot \mathrm{F}}{\mathrm{Imp} . / \mathrm{min} \ddot{U}+\mathrm{Imp} . / \mathrm{minS}}\right) \cdot 100 \quad \ldots
$$

für Trenntechniken B. und C.

Imp./min $\ddot{U}=$ Radioaktivität des Überstands-Aliquots Imp. $/$ mins $=$ Radioaktivität des Sedimentes + Restüberstand

Der Faktor $\mathrm{F}$ wird entweder durch Wägung ermittelt oder aber in Reaktionsansätzen, denen anstelle des jeweiligen Trennmittels ein identisches Volumen Wasser zugesetzt wird.
Die Radioaktivität der Proben wird mit Hilfe eines automatischen Gamma-Probenwechslers (gammaszint bf 5300) gemessen, wobei jede Probe bis zu einer Impulssumme von 10000 gezählt wird.

Die mittleren prozentualen Bindungen für die Standards werden in Abhängigkeit von den Standard-Insulin-Konzentrationen in doppelt-dekadischer Auftragung dargestellt und die ProbenAntigen-Konzen trationen über die jeweilige Standardkurve ermittelt.

Berechnungen für den Versuchsteil 4

Die Berechnung der Insulin-Konzentrationen der Proben wird für jede Trenntechnik in Verbindung mit dem jeweiligen Medium $\mathrm{a}, \mathrm{b}$ oder $\mathrm{c}$ nach drei Verfahren vorgenommen.

I. Ermittlung der Insulin-Konzentrationen aus den für die unspezifischen Bindungen nicht korrigierten Bindungswerten der Proben über die ebenfalls nicht für die unspezifischen Bindungen korrigierten Standardkurven in a, b oder $c$.

II. Ermittlung der Insulin-Konzentrationen aus den für die unspezifische Bindung UB individuell korrigierten Bindungswerten der Proben über die für die unspezifische Bindung $\mathrm{UB}_{0}$ korrigierten Standardkurven in $\mathrm{a}, \mathrm{b}$ oder $\mathrm{c}$.

III. Ermittlung der Insulin-Konzentrationen aus den für die unspezifische Bindung UB' individuell korrigierten Bindungswerten der Proben über die für die unspezifische Bindung UB' korrigierten Standardkurven in a, b oder c.

Die Korrekturen bei allen Verfahren erfolgen durch Subtraktion der in Triplikaten ermittelten mittleren unspezifischen Bindungen von den Einzelwerten der Standard-bzw. Proben-Triplikate.

\section{Ergebnisse und Diskussion}

Antigen-Antikörper-Bindung $\mathrm{B}_{0}$ und unspezifische Bindung $\mathrm{UB}_{0}$ in Abhängigkeit vom Alter des ${ }^{125} \mathrm{~J}$ Insulins bei unterschiedlichen Trenntechniken

Zur Vermeidung von Einflüssen biologischer Komponenten wird das Verhalten eines durch Radiolyse veränderten ${ }^{125} \mathrm{~J}$-Insulins in einem serumfreien Testsystem untersucht. Die verwendete Immunglobulin-Konzentration der Pufferlösung 2 ist für eine optimale Fällung 

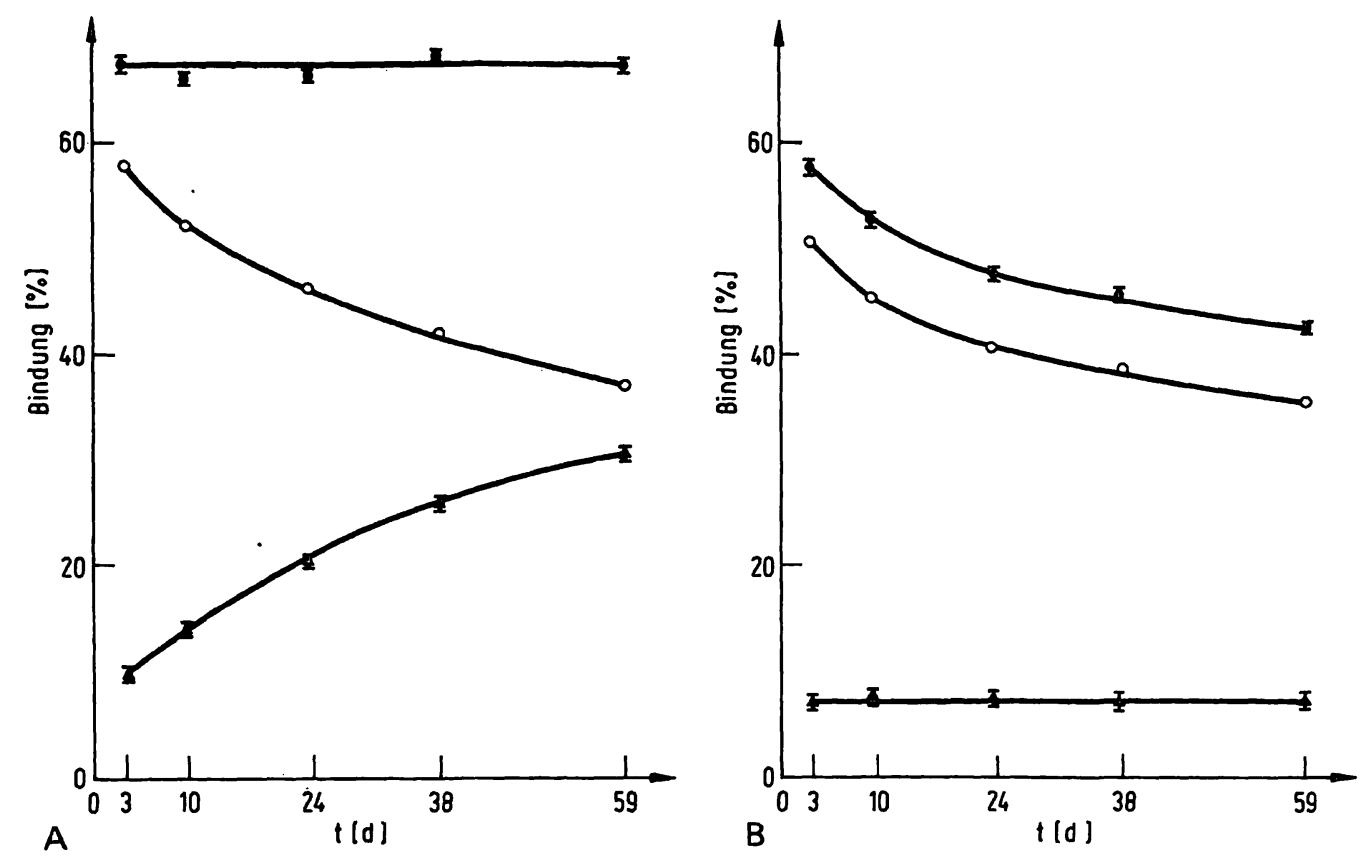

Abb. 1. \% Bindung von ${ }^{125} \mathrm{~J}$-Insulin an Insulin-Antikörper $\mathrm{B}_{\mathrm{o}}$ und \% unspezifische Bindung $\mathrm{UB}_{\mathrm{o}}$ in $A$ bhängigkeit vom Alter des $125 \mathrm{~J}$-Insulins $\overline{\mathrm{x}} \pm \mathrm{s} ; \mathrm{N}=5$.

Trennung von freiem und gebundenem Antigen: A Aktivkohle/Dextran B Natriumsulfit

Ordinaten: Bindung in $\% \bullet \mathrm{B}_{\mathrm{O}}$ Bindung in \% $\triangle \mathrm{UB}_{\mathrm{O}}$ Bindung in $\% \circ \mathrm{B}_{\mathrm{O}}-\mathrm{UB}_{\mathrm{O}}$

Abszissen: Tage nach der Markierung des ${ }^{125} \mathrm{~J}$-Insulins

des Antigen-Antikörper-Komplexes bei der Anwendung chemischer Präzipitations-Trenntechniken erforderlich (21). In der Abbildung 1 sind die Ergebnisse dargestellt. Die zu beobachtende Zunahme der unspezifischen Bindung $\mathrm{UB}_{0}$ mit dem Alter des Tracèrs bei Verwendung der Adsorptions-Trenntechnik A. ist Ausdruck für eine mangelnde Adsorption der im Verlaufe der Lagerung gebildeten Radiolyseprodukte. $\mathrm{Da}$ andererseits $\mathrm{B}_{0}$ unabhängig vom Alter des Tracers konstant bleibt, resultiert aus der Differenz $B_{0}-U B_{0}$ eine zeitabhängige Abnahme der Bindung von jeweils noch immunreaktivem Tracer. Eine Extrapolation von $\mathrm{UB}_{0}$ auf den Zeitpunkt 0 läßt erkennen, daß auch bei Verwendung eines radiolysearmen Tracers unmittelbar nach der Markierung ein Minimalwert für $\mathrm{UB}_{0}$ bestehen bleibt, der sich aus den kombinierten Effekten ergibt, die z. B. von der Adsorptions-Kapazität des Adsorbens, einer unspezifischen Adsorption än das Géfäßmaterial und einer möglichen initialen geringen radioaktiven Verunreinigung des Tracers herrühren. Bei der Trennung von freiem und gebundenem Antigen durch Präzipitation des Antigen-Antikörper-Komplexes und des noch freien Antikörper-Anteils mit Natriumsulfit (Trenntechnik B.) dagegen bleibt die unspezifische Bindung $\mathrm{UB}_{0}$ konstant, $\mathrm{B}_{0}$ nimmt mit zunehmendem Alter des Traceís ab. Bei der Korrektur von $B_{0}$ durch Subtraktion der unspezifischen Bindung $\mathrm{UB}_{0}$ findet eine Parallelverschiebung der tatsächlichen Bindungswerte in Bezug auf die unkorrigierte Bindung statt. Die im Verlaufe der Alterung des Tracers entste- henden radioaktiven Umwandlungsprodukte werden weder gefällt noch in nennenswertem Ausmaß an präzipitiertes Immunglobulin adsorbiert.

Bei der Verwendung eines nicht unmittelbar zuvor gereinigten Tracers ist die mit Hilfe der Trenntechnik A. ermittelte Bindung des ${ }^{125} \mathrm{~J}$-Insulins daher um den Betrag einer zusätzlichen vorgetäuschten Bindung erhöht, da sich die Degradationsprodukte des Tracers gegenüber dem Adsorbens wie der Antigen-AntikörperKomplex verhalten. Die Anwendung der Trenntechnik B., bei der die Radiolyseprodukte entsprechend dem freien Antigen-Anteil reagieren, verhindert einen derartigen Einfluß der Radiolyseprodukte auf den ermittelten Bindungswert.

Diese grundsätzlichen Methodik-bedingten Unterschiede lassen sich mit zwei weiteren Trenntechniken bestätigen (Tab. 1), solchen mit einem Ionenaustauscher (D.) und einem Polyalkohol (C.). Auch hierbei führt die Verwendung eines gealterten Tracers zu einer klaren Differenzierung. Der Ionenaustauscher Amberlite zeigt ein dem Adsorbens Aktivkohle/Dextran ähnliches Verhalten. Seine Bindungsfähigkeit für Radiolyseprodukte ist gegenüber derjenigen von Kohle zwar deutlich zu beobachten, sie ist jedoch unvollständig. Der hierbei ermittelte niedrigere Bindungswert $B_{0}$ mit gealtertem Tracer im Vergleich zu demjenigen mit radiolysearmem ${ }^{125} \mathrm{~J}$ Insulịn bestätigt dieses Verhalten. 
Tab. 1.\% Bindung von ${ }^{125} \mathrm{~J}$-Insulin an Insulin-Ant ikörper $\left(B_{0}\right)$ und \% unspezifische Bindung $\left(\mathrm{UB}_{0}\right)$ in $A$ bhängigkeit vom Alter des ${ }_{125} \mathrm{~J}-$ Insulins bei Anwendung der Trenntechniken A. bis $D$. $\bar{x} \pm s ; N=6$.

\begin{tabular}{|c|c|c|c|c|c|}
\hline & & \multicolumn{2}{|c|}{$\mathrm{B}_{0}(\%)$} & \multicolumn{2}{|c|}{$\mathrm{UB}_{0}(\%)$} \\
\hline & & $\overline{\mathbf{x}}$ & s & $\overline{\mathbf{x}}$ & $s$ \\
\hline A. Aktivkohle/Dextran & $\begin{array}{r}125 \mathrm{~J} \text {-Insulin } \mathrm{t}_{1} \\
" \quad \mathrm{t}_{2}\end{array}$ & $\begin{array}{l}49,5 \\
50,2\end{array}$ & $\begin{array}{l}1,5 \\
1,3\end{array}$ & $\begin{array}{l}11,6 \\
33,7\end{array}$ & $\begin{array}{l}0,1 \\
0,3\end{array}$ \\
\hline B. Natriumsulfit & $\begin{array}{ll}\# & t_{1} \\
" & t_{2}\end{array}$ & $\begin{array}{l}46,3 \\
29,6\end{array}$ & $\begin{array}{l}0,3 \\
1,5\end{array}$ & $\begin{array}{l}9,0 \\
8,5\end{array}$ & $\begin{array}{l}1,0 \\
0,9\end{array}$ \\
\hline C. Polyethylenglycol 4000 & $\begin{array}{ll}\# & t_{1} \\
\# & t_{2}\end{array}$ & $\begin{array}{l}48,9 \\
31,1\end{array}$ & $\begin{array}{l}1,7 \\
0,7\end{array}$ & $\begin{array}{l}8,8 \\
7,5\end{array}$ & $\begin{array}{l}0,9 \\
0,6\end{array}$ \\
\hline D. Amberlite CG $400 \mathrm{I}$ & $\begin{array}{ll}" & t_{1} \\
" & t_{2}\end{array}$ & $\begin{array}{l}44,9 \\
37,6\end{array}$ & $\begin{array}{l}0,2 \\
0,9\end{array}$ & $\begin{array}{r}8,8 \\
16,1\end{array}$ & $\begin{array}{l}0,3 \\
1,2\end{array}$ \\
\hline
\end{tabular}

Wird zur Trennung Polyethylenglycol 4000 verwendet, so können dem Natriumsulfit analoge Verhältnisse beobachtet werden. Die Werte für die unspezifische Bindung $\mathrm{UB}_{0}$ sind unabhängig vom Alter des Tracers konstant, $B_{0}$ sinkt mit zunehmender Alterung.

\section{Antigen-Antikörper-Bindungen $\mathrm{B}$ und unspezifische Bindungen $\mathrm{UB}$ und $\mathrm{UB}^{\prime}$ bei konstanter und bei variabler Tracer-Konzentration}

Zur Untersuchung der Abhängigkeit der unspezifischen Bindung UB vom Antigen-Antikörper-Verhältnis wird außer einer Standardkurve die zu jeder Standard-Insulin-Konzentration gehörende unspezifische Bindung $\mathrm{UB}_{\mathrm{St}}$ und außerdem UB' bei einer Standard-Insulin-Konzentration von $500 \mathrm{E} / \mathrm{l}$ ermittelt (Abb. 2). Wie die Ergebnisse zeigen, sind auch die Extremwerte innerhalb

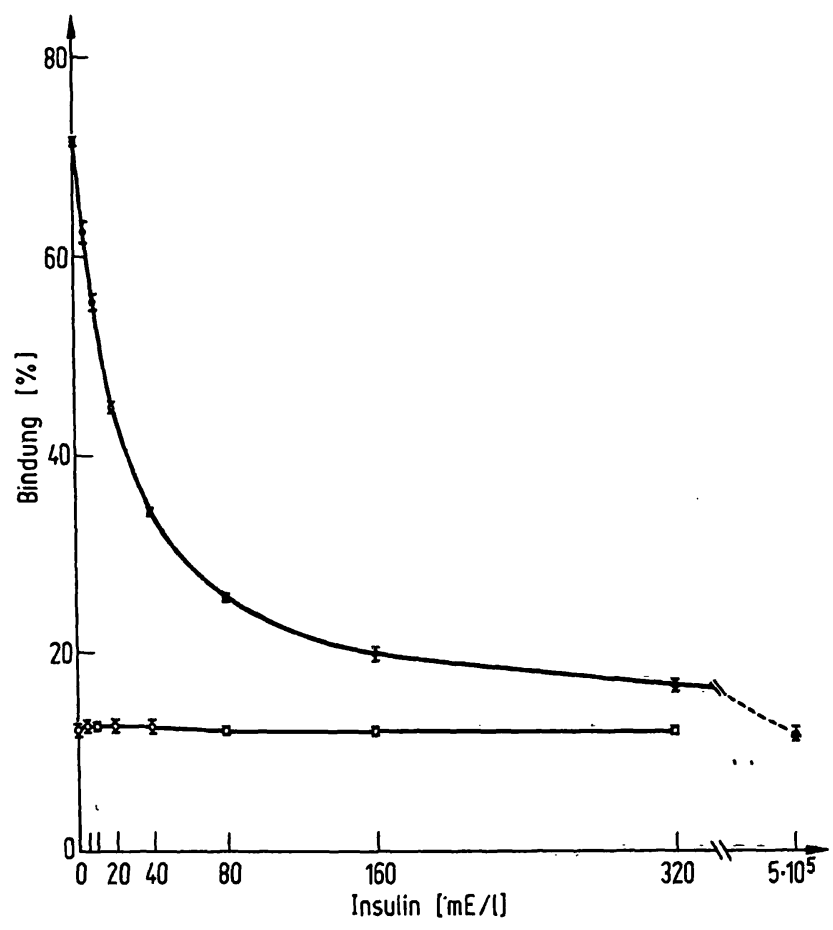

Abb. 2. \% Bindung BSt $(\bullet)$ und \% unspezifische Bindung UB $\mathrm{Bt}_{\mathrm{St}}$ (०) für acht Insulin-Konzentrationen; \% unspezifische Bindung UB' bei $500 \mathrm{E} / 1$ Insulin ( $\Delta) \quad \overline{\mathrm{x}} \pm s ; N=3$ Trennmittel: Natriumsulfit des untersuchten Konzentrationsbereiches, d. h. die individuellen unspezifischen Bindungen $\mathrm{UB}_{S \mathrm{St}}$ am $\mathrm{An}$ fang und am Ende der Standardkurve, nicht voneinander $\mathrm{zu}$ unterscheiden. Weiterhin wird beobachtet, daß UB' von gleicher Größe und nicht von $U^{\prime} B_{S t}$ differenzierbar ist.

Da Unterschiede zwischen Anfangs- und Endteil der Standardkurve nur aufgrund der relativen Konzentrations-Unterschiede von gebundenem und freiem Traceranteil bestehen und daher auch nur unterschiedlich hohe Absolutwerte eines dieser Tracerkompārtimente einen Einfluß auf die Höhè der unspezifischen Bindungen haben können (4), wird auch das Verhalten von $\mathrm{B}_{0}$, UB und $\mathrm{UB}^{\prime}$ in Abhängigkeit von der Tracerkonzentration untersucht (Abb. 3). Hierbei zeigt sich, daß weder die Tracerkonzentration noch ihre relative Vertei-

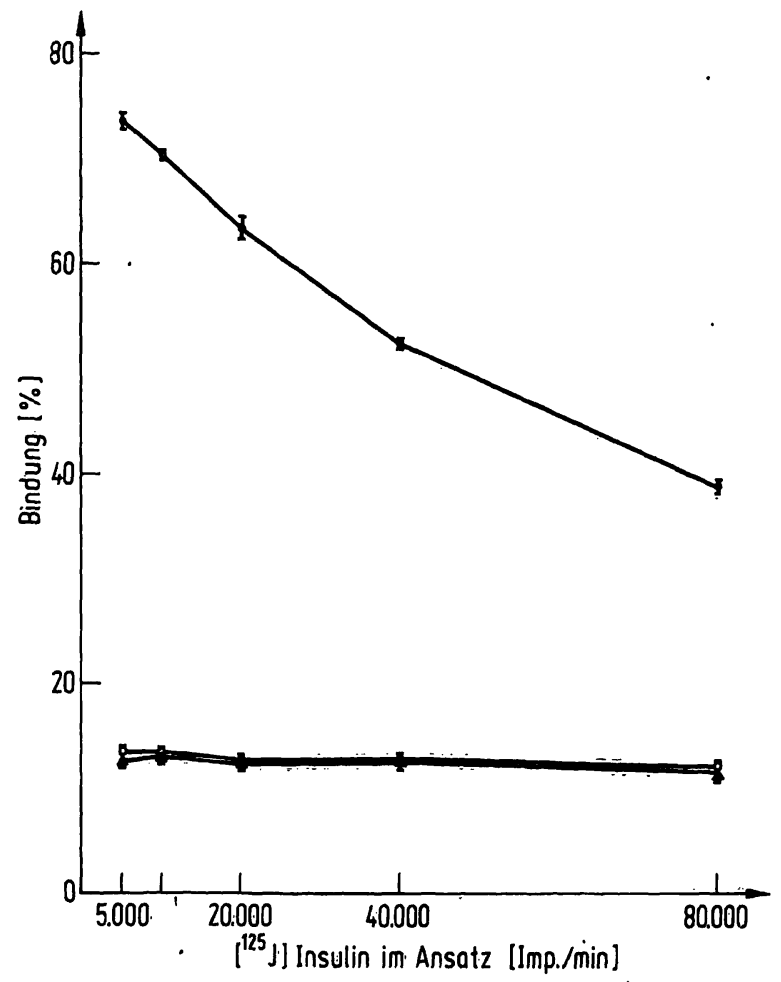

$A b b$. 3. \% Bindung $B_{o}(\bullet)$ und \% unspezifische Bindungen $\mathrm{UB}_{0}$ (o) und $\mathrm{UB}^{\prime}$ bei $500 \mathrm{E} / \mathrm{l}$ Insulin ( $(\Delta)$ in Abhängigkeit von der ${ }^{125}$ J-Insulin-Konzentration $\bar{X} \pm s ; N=3$ Trennmittel: Natriumsulfit 
lung auf den freien und den gebundenen Anteil einen Einfluß auf die Höhe der unspezifischen Bindungen UB und $\mathrm{UB}^{\prime}$ hat. Die Konstanz von $\mathrm{UB}_{\mathrm{St}}$, ihre Identität mit $\mathrm{UB}^{\prime}$ und die Unabhängigkeit von UB und UB' von der relativen Verteilung des Tracers sowie seiner absoluten Menge legalisieren daher die uniforme subtraktive Korrektur aller Standardwerte für die anhand eines beliebigen Standards ermittelte unspezifische Bindung UB $\mathrm{B}_{\mathrm{St}}$ oder auch für UB'.

Bindung von ${ }^{125} \mathrm{~J}$-Insulin und unspezifische Bindungen UB und UB' in Anwesenheit endogenen Antikörpers

Anhand von Versuchen zur Bestimmung der Insulin-Konzentrationen in Seren von Diabetikern kann der störende Einfluß des endogenen Antikörpers auf den Test demonstriert werden (Tab. 2). Während die Antigen-Antikörper-Bindung des zum Vergleich untersuchten insulinarmen Humanserums mit $48,9 \%$ in Höhe von $B_{0}$ $(49,8 \%)$ der Standardkurve liegt, zeigen beide Seren, $S_{1}$ mit $75,8 \%$ und $S_{2}$ mit $74,6 \%$, hierbei ungewöhnlich hohe Bindungswerte. Auch die unspezifischen Bindungen für diese beiden Seren indizieren eine Teststörung. Die ohne Zusatz exogenen Antiserums ermittelten unspezifischen Bindungen UB sind für $S_{1}$ und $S_{2}$ nahezu identisch mit den Bindungswerten, die in Gegenwart des im Test vorgelegten exogenen Antiserums gefunden werden. Hieran wird deutlich, daß die Antigen-AntikörperBindungen in derartigen Seren fast ausschließlich durch den endogenen Antikörper bestimmt werden. Der Versuch, die unspezifischen Bindungen $\mathrm{UB}^{\prime}$ für diese Seren durch Zusatz von $100 \mathrm{E} / \mathrm{l}$ zu ermitteln, senkt die Bindungswerte jedoch nur geringfügig, woraus hervorgeht, daß infolge des hohen endogenen Antikörper-Gehaltes. die zugesetzte Insulinmenge nicht ausreicht, um eine vollständige kompetitive Verdrängung des markierten Antigens vom Antikörper zu bewirken. Durch eine Un-

Tab. 2. Der Einfluß von Serumproben mit endogenem InsulinAntikörper auf die Bindung des ${ }^{125} \mathrm{~J}$-Insulins an exogenen Insulin-Antikörper. Bindung in $\% ; \bar{x} \pm s ; N=3$.

\begin{tabular}{|c|c|c|c|}
\hline & & \multicolumn{2}{|c|}{ Bindung (\%) } \\
\hline & & $\dot{\bar{x}}$ & s \\
\hline \multirow[t]{2}{*}{ Standardkurve } & $\begin{array}{r}0 \mathrm{mE} / 1 \\
5 \mathrm{mE} / 1 \\
10 \mathrm{mE} / 1 \\
20 \mathrm{mE} / 1 \\
40 \mathrm{mE} / 1 \\
80 \mathrm{mE} / 1 \\
160 \mathrm{mE} / 1\end{array}$ & $\begin{array}{l}49,8 \\
44,1 \\
40,4 \\
33,9 \\
26,3 \\
20,1 \\
15,8\end{array}$ & $\begin{array}{l}0,6 \\
0,3 \\
0,4 \\
0,3 \\
0,5 \\
0,2 \\
0,3\end{array}$ \\
\hline & $\begin{array}{l}\text { UB } \\
\text { UB' }^{\prime}\end{array}$ & $\begin{array}{l}9,2 \\
8,4\end{array}$ & $\begin{array}{l}0,02 \\
0,7\end{array}$ \\
\hline insulinarmes Humanserum & $\begin{array}{l}\text { UB } \\
\text { UB' }\end{array}$ & $\begin{array}{r}48,9 \\
9,8 \\
9,7\end{array}$ & $\begin{array}{l}0,5 \\
0,9 \\
0,6\end{array}$ \\
\hline Diabetiker-Serum $S_{1}$ & $\underset{\mathbf{U B}^{\prime}}{\mathrm{UB}}$ & $\begin{array}{l}75,8 \\
70, \hat{2} \\
38,0\end{array}$ & $\begin{array}{l}0,3 \\
0,9 \\
0,4\end{array}$ \\
\hline Diabetiker-Serum $S_{2}$ & $\begin{array}{l}\text { UB } \\
\text { UB' }^{\prime}\end{array}$ & $\begin{array}{l}74,6 \\
75,2 \\
50,4\end{array}$ & $\begin{array}{l}0,7 \\
0,6 \\
0,2\end{array}$ \\
\hline
\end{tabular}

tersuchung des Zusammenhanges von UB' mit der Konzentration unmarkierten Antigens kann anhand der Resultate für das Serum $S_{3}$ (Abb. 4) gezeigt werden, daß selbst der Zusatz extrem großer Antigenmengen nicht immer ausreicht, um einen Approximativwert der unspezifischen Bindung UB' zu ermitteln.

Seit Hirata et al (13) 1970 erstmalig über die Existenz von Insulin-Autoantikörpern berichteten und weiterhin eine Reihe ähnlicher Beobachtungen beschrieben wurden $(12,14-20)$, ist es wünschenswert, innerhalb der Routine-Insulin-Bestimmung die Möglichkeit zu haben, einen derartigen Einfluß auf den Test erkennen zu können. Die für jede Serum- oder Plasmaprobe ermittelte individuelle unspezifische Bindung UB ist hierzu geeignet und sie stellt einen eindeutigen Indikator dar, daß für solche Seren die Ermittlung der Insulin-Konzentration durch direkte Messung nicht anwendbar ist.

Die Wiederfindung von Insulin in Serum unter Verwendung von Standardkurven in unterschiedlichen Medien und der Einfluß der unspezifischen Bindungen auf die Richtigkeit der Ergebnisse

Aufgrund von 3 Standard-Medien und 3 Trenntechniken bestehen neun Varianten zur experimentellen Ermittlung der Insulin-Konzentrationen der 9 Proben. Außerdem werden jeweils die Verfahren I., II. und III. zur Berechnung angewandt. Für jede dieser insgesamt 27 Möglichkeiten wird zunächst durch Korrelation der Daten: der Probe zugesetzte $\mathrm{mE} / \mathrm{l}$ Insulin und gefundene $\mathrm{mE} / \mathrm{l} \mathrm{In}$ sulin in der Probe der jeweilige Basalwert des Serums ermittelt. Die Daten: eingesetzte $\mathrm{mE} / \mathrm{l}$ Insulin (Basalwert + zugesetzte $\mathrm{mE}$ Insulin) und in der Probe gefundene $\mathrm{mE} / \mathrm{l}$ Insulin werden korreliert; mit ihrer Hilfe werden die mittleren Wiederfinderaten berechnet.

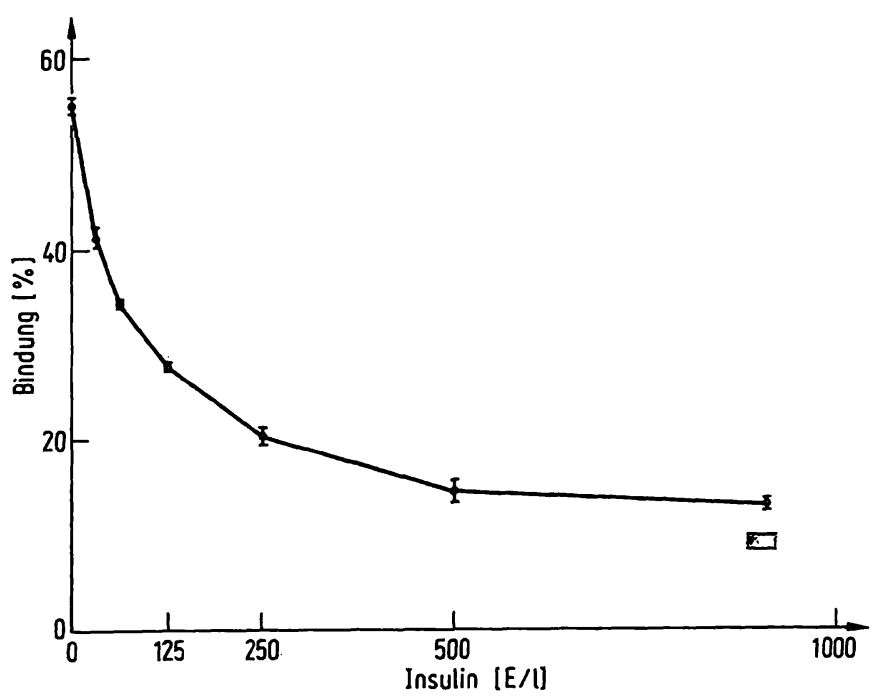

Abb. 4. \% Bindung von ${ }^{125} \mathrm{~J}$-Insulin bei Anwesenheit eines endogenen Antikörpers $\left(S_{3}\right)$ in Abhängigkeit von der Menge an zugesetztem Standard-Insulin $\bar{x} \pm s ; N=3$. Ordinate: gebundenes ${ }^{125} \mathrm{~J}$-Insulin in \%

Abszisse: Standard-Insulin in E/l dem Serum zugesetzten Insulins

: Bereich der unspezifischen Bindung UB' für ein insulinarmes Serum 


\section{Standard-Medium a}

Abbildung 5 zeigt die Wiederfindung von Insulin exemplarisch für die Kombination: Standard-Medium a, Trenntechnik A., Berechnungsverfahren I., II. und III. Die Korrelationsdaten und die mittleren Wiederfinderaten hierfür und für die Trenntechniken B. und C. sind in der Tabelle 3 aufgefuhrt. Bei der Verwendung einer Lösung von $20 \mathrm{~g} / \mathrm{l}$ Immunglobulin vom Rind in Puffer-

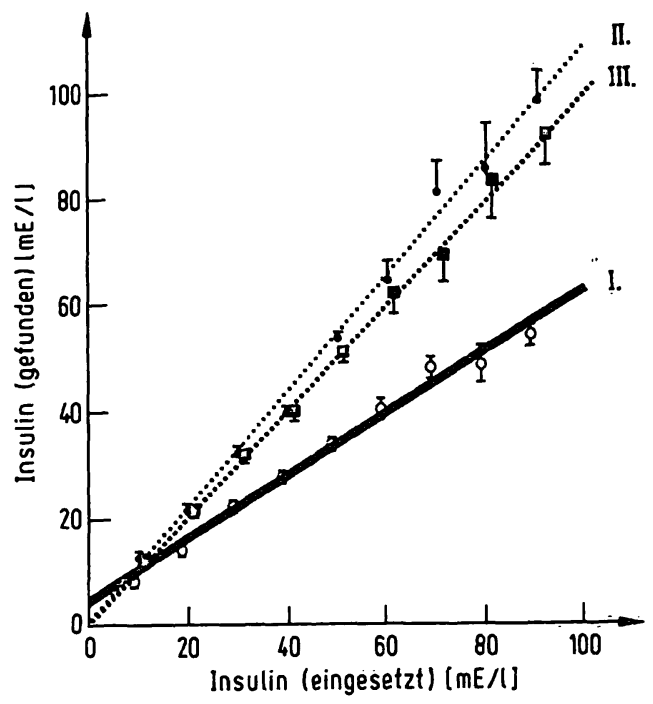

Abb. 5. Wiederfindung von Insulin in Serum Standards in Medium a; Trenntechnik A.; Berechnungsverfahren I., II. und III.

Ordinate: $\mathrm{mE} / \mathrm{l}$ Insulin gefunden $\quad \overline{\mathrm{x}} \pm s ; \mathrm{N}=3$

Abszisse: $\mathrm{mE} / 1$ Insulin eingesetzt lösung 1 - Medium a - als Milieu zur Herstellung der Standards werden bei der Ermittlung der Serum-InsulinKonzentrationen unter Verwendung von Aktivkohle/ Dextran und ohne Berücksichtigung der unspezifischen Bindungen zu niedrige Werte gefunden, für die Trennmittel Natriumsulfit und Polyethylenglycol zu hohe Werte. Werden dagegen die Berechnungsverfahren II. oder III. angewandt, so kann für jede der drei Trenntechniken eine ausreichende Wiederfindung beobachtet werden.

\section{Standard-Medium b}

Auch die Ergebnisse, die bei Verwendung des insulinarmen Humanserums Fluinorm - Medium b - als Standard-Milieu resultiêren, zeigen, daß unabhängig von der Art der Trenntechnik eine ausreichende Wiederfindung erst erreicht wird, wenn eine individuelle Korrektur der gemessenen Bindungswerte für die unspezifischen Bindungen UB oder UB' vorgenommen wird (Tab. 4).

\section{Standard-Medium c}

Wird in der Bezugs-Standardkurve eine Milieu-Zusammensetzung von $50 \mathrm{~g} / \mathrm{l}$ Humanalbumin und $20 \mathrm{~g} / \mathrm{l}$ Immunglobulin vom Rind in Pufferlösung 3 - Medium c - gewählt (Tab. 5), so kaṇn nur bei der Verwendung von Natriumsulfit als Trennmittel eine optimale Wiederfindung beobachtet werden. Diese Kombination stellt den einzigen Fall aller untersuchten Möglichkeiten dar, bei dem auch ohne Berücksichtigung der unspezifischen Bindungen die Wiederfindung mit den nach Korrektur

Tab. 3. Korrelationen von eingesetzten $(x)$ und gefundenen $(y)$ Serum-Insulin-Konzentrationen und mittlere Wiederfinderaten MW in \% für die Trenntechniken A., B. und C.; Berechnungsverfahren I., II. und III.; Medium a.

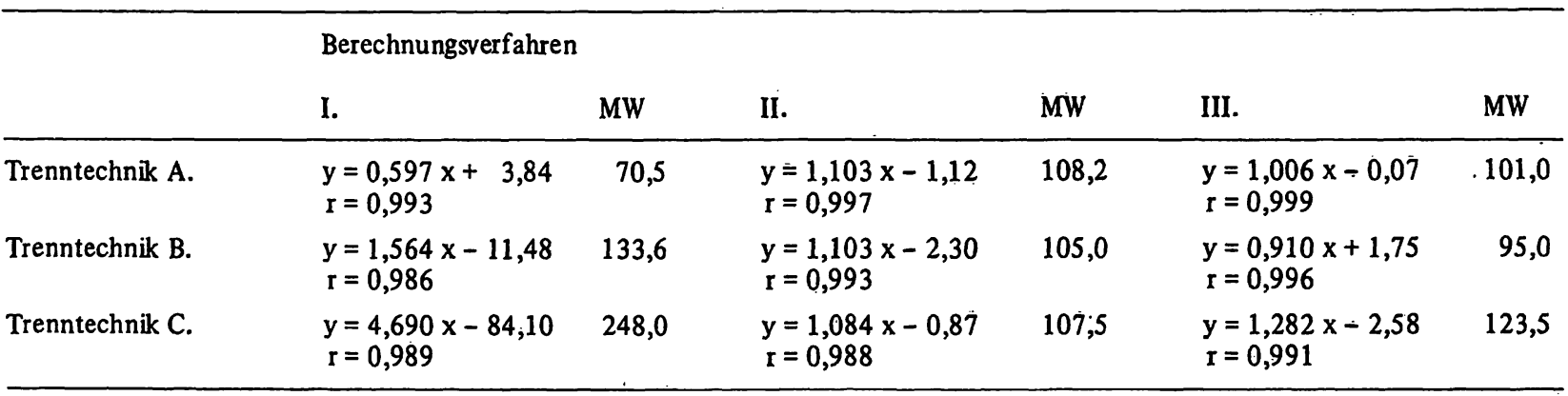

Tab. 4. Korrelationen von eingesetzten ( $x$ ) und gefundenen ( $y$ ) Serum-Insulin-Konzentrationen und mittlere Wiederfinderaten MW in \% für die Trenntechniken A., B. und C.; Berechnungsverfahren I., II. und III.; Medium b.

\begin{tabular}{|c|c|c|c|c|c|c|}
\hline \multirow{2}{*}{. } & \multicolumn{6}{|l|}{ Berechnungsverfahren } \\
\hline & I. & MW & II. & MW & III. & MW \\
\hline Trenntechnik A. & $\begin{array}{l}y=0,455 x+4,81 \\
r=0,991\end{array}$ & 60,1 & $\begin{array}{l}y=1,157 x-2,34 \\
r=0,996\end{array}$ & 109,7 & $\begin{array}{l}y=1,130 x-1,92 \\
r=0,999\end{array}$ & 107,8 \\
\hline Trenntechnik B. & $\begin{array}{l}y=3,102 x-5,86 \\
r=0,970\end{array}$ & 366,0 & $\begin{array}{l}y=1,025 x-0,46 \\
x=0,993\end{array}$ & 101,1 & $\begin{array}{l}y=1,005 x-0,10 \\
r=0,996\end{array}$ & 100,9 \\
\hline Trenntechnik C. & $\begin{aligned} y & =7,450 x-285,1 \\
r & =0,970\end{aligned}$ & 207,0 & $\begin{array}{l}y=1,129 x=1,75 \\
x=0,992\end{array}$ & 109,1 & $\begin{array}{l}y=1,250 x-2,94 \\
r=0,993\end{array}$ & $119 ; 0$ \\
\hline
\end{tabular}


Tal2. 5. Korrelationen von eingeselzten $(x)$ und gofundenon $(y)$ Sorum-lnsulin-Konzentrationen und mittlore Wioderlinderaten MW in "\% flir die Trenntechniken A., B. und C.; Berechnungsverfaliren l., II. IInd III.; Medium c.

Berechnungsverfahren

\begin{tabular}{|c|c|c|c|c|c|c|}
\hline & I. & MW & II. & $M W$ & III. & $M W$ \\
\hline Trenniechnik A. & $\begin{array}{l}y=0,760 x+2,11 \\
r=0,993\end{array}$ & 84,4 & $\begin{array}{l}y=1,359 x-2,77 \\
r=0,989\end{array}$ & 133,1 & $\begin{array}{l}y=1,167 x-1,39 \\
r=0,996\end{array}$ & 116,4 \\
\hline Trenntechnik B. & $\begin{array}{l}y=0,993 x+0,14 \\
r=0,996\end{array}$ & 99,7 & $\begin{array}{l}y=0,99() x-1,05 \\
r=0,993\end{array}$ & 97,7 & $\begin{array}{l}y=0,965 x+0,58 \\
r=0,996\end{array}$ & $9 K, 3$ \\
\hline Trenntechnik C. & $\begin{array}{l}y=2,597 x-8,96 \\
r=0,988\end{array}$ & 248,9 & $\begin{array}{l}y=1,335 x-4,40 \\
r=0,991\end{array}$ & 122,7 & $\begin{array}{l}y=1,1,10 x-1,58 \\
r=0,994\end{array}$ & $|1|, 0$ \\
\hline
\end{tabular}

für die unspezifischen Bindungen erhaltenen Daten iibereinstimmt. Bei einem Vergleich der Werte, die aus der Aktivkohle/Dextran- und der PolyethylenglycolTrennung resultieren, wird bestăligl, daß olnne Berücksiclıtigung der unspezifischen Bindungen die Adsorp. tions-Trenntechnik wiederum zu niedrige Werte, die Prizipitations-Trenntechnik zu hohe Werte ergibt, daß die Korreklur fiir die unspezifisclien Bindungen hier aber nicht zu einer befriedigenden Wiederfindung fülirt.

\section{SchluRdiskussion und Folgerungen}

Beim radioimmunologischen Test lassen sich AnalysenLeerwerte in unterschiedlicher Weise ermitteln; zudem sind die unspezifischen Bindungen von Standards und Proben zumeist nicht identisch und auch für die unspezifischen Bindungen der Standards selbst wurcle eine Abhängigkeit vom Antigen/Antikörper-Verhältnis nicht ausgeschlossen. Insofern ist die Problemalik der Leerwerte heim Radioimmunoassay komplexer als bei anderen Analysen-Verfahren und eine Untersuchung zur unspezifischen Bindung sollte zunächst exemplarisch für ein Antigen erfolgen, wobei nur eine stufenweise Beschreibung der folgenden Zusammenhänge Empfehlungen zur Behandlung der unspezifischen Bindungen erwarten lassen kann:

1. Ermittlung der unspezifischen Bindungen UB und $U B^{\prime}$ für Standards und deren Beeinflussung

2. Einflüsse von $U B$ und $U^{\prime} B^{\prime}$ auf die Standardkurve

3. Verhalten der unspezifischen Bindungen UB und UB' für Seren, die eine Teststörung bewirken und ihre Bedeutung für die Brkennung von abnormen Serumprohen

4. Einfluß der individuellen unspezifischen Bindungen UB und UB' für Standards und Proben auf die Riclstigkeit der Ergebnisse

Die für das Insulin beschriebenen Zusammenhänge lassen sich vermutlich nicht uneingeschränkt auf weitere Antigene übertragen, da die physikal isch-chemischen Antigen-Eigenschaften ein unterschiedliches Verhalten ihrer unspezifischen Bindungen nicht ausschließen. Unabhängig hiervon kann jedoch auch für sle die grund- sïlzliclıe Emprehlung zur Brrmiltlung der inclividuellen unspezifisclien Binclungen gelten, insbesonclere dann, wenn das Auftreten von Antikörpern, Aulu-Antikörpern oder anderer Siörfikloren in der Probe wahrscheinlich ist.

Die in der radioimmunologischen Praxis zur Bestimmung der Konzentration eines Anligens verwendelen Testaufbauten untersclieiden sich melhodisch sowohl bei den kommerziellen Testkombinalionen als auch hei den laborinternen Tesis neben der Verwendung individueller $\mathrm{Antiseren}$ und Inkubations-Bedingungen im Wesenllichen in der Arl der Trennleclinik und der Zusammensetzung der Medien für die Slandards.

Die hier in unterschiedlicher Kombinalion von Trenntechnik und Standard-Medium erzielten Resullate zeigen, daß bei sonst iden lischen Tes/bedingungen weder eine Anderung des Standard-Mediums bei vorgegebener Trenntechnik noch eine Änderung der Trenntechnik für ein vorgegebenes Standard-Medium zu ciner oplimalen Wiederfind ung fïlırl, wenn die unspezifischen Bindungen von Probe und Standard unberïcksichtigl bleiben. So werden bei der Adsorptions-Trenn technik uIIablïngig vom Standard-Medium zu niedrige Werte fir die erwarteten Proben-An tigen-Konzen trationen gefunden, bei den chemischen Präzipitations-Trenntechniken zu hohe Werte. Von den insgesamt neun der in diesem Zusammenhange untersuchıten Möglichkeiten fihhrt nur eine (Standard-Medium c, Trenmmittel Natriumsulfit) ohne Korrek tur für die unspezifischen Bindungen zu einer ausreichend guten Wiederfindung, die aber unter Beriicksichtigung der zahlreiclien praktizierten Modifikationen in den Testaufhaulen als zufillig opt imale Kombination anzuschen ist. Die bis auf diese Ausnahme generoll mangelnde oder übermiißig hohe Wiederfindung ist vorwiegend eine Folge der Differenzen in den unspezifischen Bindungen von Standards und Proben. Mil der Adsorplions-Trenntechnik werden fïr jedes der drei Standard-Medlen a, b und $\mathrm{c}$ niedrigere unspezilisclie Bindungen gegentibor den unspezifischen Bindungen der Prohen beobachlet. Die Bindungswerte fir die Proben liegen daher bei Vernachlässigung der unspezifischen Bindungen relativ zu hooh, die ermittelten Konzentrationswerte zu niedrig. 
Die bei Anwendung der chemischen PräzipitationsTrenntechniken ermittelten unspezifischen Bindungen zeigen ein entgegengesetztes Verhalten. Durch Fällung mit Natriumsulfit resultieren bei den Standard-Medien $a$ und $b$ höhere, bei dem Medium $c$ identische unspezifische Bindungen im Vergleich zu den Proben. Die Fällung mit Polyethylenglycol 4000 führt für jedes der Standard-Medien $\mathrm{a}, \mathrm{b}$ und $\mathrm{c}$ zu höheren unspezifischen Bindungen gegenüber denjenigen der Proben. Bei den chemischen Präzipitations-Trenntechniken werden daher die Bindungswerte für die Proben relativ zu niedrig gefunden, die ermittelten Konzentrationswerte zu hoch.

Diese Differenzen werden sowohl für die Adsorptionsals auch für die Präzipitations-Trenntechniken bei der Mehrzahl der untersuchten Kombinationen dadurch aufgehoben, daß die individuellen unspezifischen Bindungen bei der Berechnung der Proben-Antigen-Konzentrationen berücksichtigt werden.

Hierbei besteht kein signifikanter Unterschied zwischen der Korrektur furr die ohne Antiserum ermittelte unspezifische Bindung UB und der in Gegenwart von Antiserum und Antigen-Überschuß ermittelten unspezifischen Bindung $\mathrm{UB}^{\prime}$. Die individuelle Korrektur für UB oder UB' bewirkt in jedem Falle eine deutliche Verbesserung der Wiederfinderaten.

Im allgemeinen werden bei radioimmunologischen Tests individuelle unspezifische Bindungen nur außerordentlich selten ermittelt. Dagegen wird häufiger ein Verfahren angewandt, bei dem eine unspezifische Bindung für einen Standard ermittelt und dann für Standards und
Proben in gleicher Weise zur Korrektur herangezogen wird; die individuellen Unterschiede zwischen Probenund Standard-Medium jedoch müssen den Erfolg eines derartigen Vorgehens in Frage stellen.

Neben der Bedeutung der individuellen unspezifischen Bindungen als Indikator zur Erkennung von Antikörpern oder Auto-Antikörpern bei der Insulin-Bestimmung lassen die Resultate aus den Wiederfindeversuchen vermuten, daß eine Notwendigkeit zur Verwendung der individuellen unspezifischen Bindungen als Korrekturgröße im Sinne des Analysen-Leerwertes bei der Berechnung von Insulin-Konzentrationen in Serumproben besteht aufgrund der Differenzen, die die unspezifischen Bindungen von Standards und Proben nahezu regelmäßig zeigen.

Die für die Insulin-Bestimmung aus diesen Gründen erhobene Forderung nach Berücksichtigung der individuellen unspezifischen Bindungen bleibt u. E. auch für weitere radioimmunologische Tests bestehen, für deren Antigen zwar Antikörper oder Auto-Antikörper unbekannt sind, bei denen aber Medien-Unterschiede zwischen Probe und Standard unvermeidbar sind.

In Hinsicht auf die notwendige Standardisierung und Qualitätskontrolle beim Radiọmmunoassay können die Resultate dieses Modellversuchs einen Hinweis geben, daß die individuelle unspezifische Bindung auch bei weiteren radioimmunologischen Testverfahren eine die Richtigkeit der Ergebnisse beeinflussende Größe darstellen kann.

\section{Literatur}

1. Marschner, I., Bottermann, P., Erhardt, F., Linke, R., Löffler, G., Maier, V., Schwandt, P., Vogt, W. \& Scriba, P. C. (1974), Horm. Metab. Res. 6, 293-296.

2. Marschner, I., Biro, G., Erhardt, F. W., Scriba, P. C. \& Weinges, K. (1975), Informationsblatt der Deutschen DiabetesGesellschaft Nr. 3, 1-9.

3. Standardization of Radioimmunoassay Procedures (1974), Int. J. Appl. Radiat. Isot., 2.5, 145-160.

4. Ekins, R. \& Newman, B. (1970), Karolinska symposia on research methods in reproductive endocrinology, 2nd symposium, 11-30.

5. Cerceo, E. \& Cipriano, A. E. (1972), Clin. Chem. 18, 539543.

6. Holtzman, J. L., Shafer, R. B. \& Erickson, R. R. (1974), Clin. Chem. 20, 1194-1198.

7. Voshall, D. L., Hunter, L. \& Grady, H. J. (1975), Clin. Chem. 21, 402-406.

8. Burnett, G. H., Conklin, R. L., Wasson, G. W. \& MacKinney, A. A. (1973), Clin. Chem. 19, 725-726.

9. Hofman, L. F. (1973), Clin. Chem. 19, 1413-1414

10. Anggard, E. E., Chew, L. F. \& Kalman, S. M. (1972), N. Engl. J. Med. 287, 935.

11. Yalow, R. S. \& Berson, S. A. (1970), Gastroenterology 58, $1-14$.
12. Kawazu, S., Kanazawa, Y., Kajinuma, H., Miki, E., Kuzuya, T. \& Kosaka, K. (1975), Diabetologia 11, 169-173.

13. Hirata, Y., Ishizu, H., Ouchi, N., Motomura, S., Abé, M., Hara, Y., Wakasugi, H., Takahashi, İ., Takano, H., Tanaka, M., Kawano, H. \& Kanasaki, T. (1970), J. Japan. Diab. Soc. $13,312-319$.

14. Hirata, Y. \& Arimachi, M. (1972), J. Japan. Diab. Soc. 15, $187-192$.

15. Folling, I. \& Norman, N. (1972), Diabetes 21, 814-826.

16. Nakagaiva, S., Suda, S., Kudo, M. \& Kawasaki, M. (1973), Diabetologia 9, 367-375.

17. Ohneda, A., Matsuda, K., Sato, M., Yamagata, S. \& Sato, T. (1974), Diabetes 23, 41-50.

18. Hirata, Y., Nishimura, H., Tominaga, M., Oguchi, T. \& Nakamura, Y. (1972), J. Japan. Diab. Soc. 15, 336-342.

19. Sato, T., Saito, T., Honma, K., Ono, H., Yasuda, K. \& Torikai, T. (1972), J. Japan. Diab. Soc. 15, 176.

20. Nakano, M., Ito, M., Yamamoto, T. \& Hamazaki, T. (1974), J. Japan. Diab. Soc. 17, 143.

21. Dwenger, A., Zick, R., Friedel, R. \& Trautschold, I. (1976), Z. Anal. Chem. 279, 108-109.

Dr. A. Dwenger Inst. f. Klin. Biochem. u. Physiol. Chem. d. MHH

Karl-Wiechert-Allee 9

3000 Hannover 61 